\title{
Microfluidic platform for controlling the differentiation of embryoid body
}

\author{
Wai-To Fung ${ }^{a}$, Ali Beyzavi ${ }^{b}$, Patrick Abgrall ${ }^{b}$, Nam-Trung Nguyen ${ }^{*}$ and Hoi-Yeung Li $*^{a}$ \\ Receipt/Acceptance Data [DO NOT ALTER/DELETE THIS TEXT] \\ Publication data [DO NOT ALTER/DELETE THIS TEXT] \\ DOI: 10.1039/b000000x [DO NOT ALTER/DELETE THIS TEXT]
}

\begin{abstract}
Embryonic stem (ES) cells are pluripotent cells, which can differentiate into any cell types. This cell type has often been implicated as an eminent source of renewable cells for tissue regeneration and cellular replacement therapies. Studies on manipulation of the various differentiation pathways have been at the 10 forefront of research. There are many ways in which ES cells can be differentiated. One of the most common techniques is to initiate the development of embryoid bodies (EBs) by in vitro aggregation of ES cells. Thereafter, EBs can be induced to undergo differentiation into various cell lineages. In this report, we present a microfluidic platform using biocompatible materials, which is suitable for culturing $\mathrm{EB}$. The platform is based on a Y-channel device with two inlets for two different culturing media. An EB is 15 located across both streams. Using the laminar characteristics at low Reynolds number and high Peclet numbers, we have induced cell differentiation on a half of the EB while maintaining the other half in uninduced stages. The results prove the potential of using microfluidic technology for manipulation of EBs and ES cells in tissue engineering.
\end{abstract}

Keywords: embryoid bodies, differentiation, microfluidics, laminar flow, diffusion

\section{${ }_{20}$ Introduction}

Embryonic stem (ES) cells are derived from the inner cell mass of the blastocyst of a developing embryo ${ }^{1}$. They are pluripotent cells which could virtually give rise to any cell type including neural cells, cardiomyocytes and hepatocytes 25 through cellular differentiation ${ }^{2}$. ES cells are self-renewable and for that reason, they have often been implicated as an eminent source of renewable cells for tissue regeneration and cellular replacement therapies. Hence, it is not surprising that the control and manipulation of the various differentiation 30 pathways in ES cells have been at the forefront of research.

There are many ways to differentiate ES cells. One of the most common technique is to initiate the development of embryoid bodies (EBs) by in vitro aggregation of ES cells ${ }^{3,4}$. Thereafter, EBs can be induced to undergo differentiation into 35 the various cell lineages ${ }^{4-7}$. To date, no attempt was ever made to sub-differentiate a single EB into more than one lineage. This is mainly compounded by the limitations of conventional culturing techniques, which do not permit an EB to be induced by more than one differentiation media at the same time.

40 Microfabricated culturing systems can circumvent this limitation and permit the control of the differentiation process by manipulating the environment of the culture in both 'time and space', which is almost impossible to achieve using conventional culturing protocols. The behaviour of fluids at

\footnotetext{
${ }^{a}$ Division of Molecular and Cell Biology, School of Biological Sciences, Nanyang Technological University, 60 Nanyang Drive, Sciences, Nanyang
Singapore 637551

b School of Mechanical and Aerospace Engineering, Nanyang Technological University, 50 Nanyang Avenue, Singapore, 639798

*E-mail: hyli@ntu.edu.sg; mntnguyen@ntu.edu.sg

$\dagger$ Electronic Supplementary Information (ESI) available:. See http://dx.doi.org/10.1039/b000000x/
}

45 the microscale is different from that in macroscale. For microfluidic systems some gripping and counterintuitive properties appear. When two fluids streams come together in a microchannel, a parallel laminar flow can be generated without turbulence ${ }^{8}$. The Reynolds number, which represents 50 the ratio between inertial force and viscous force, is in microscale often less then unity. At these low Reynolds numbers, the Peclet number, which represents the ratio between advection and diffusion, are still in the order of 1000 . Thus a microchannel would allow two liquids to follow side 55 by side without major intermixing. This unique property of liquid in microchannel enables possibilities and new tools for biological research.

With recent advance on design and fabrication of microfluidic platform, microfluidic platform has been applied ${ }_{60}$ in research areas including stem cell and developmental biology. Manipulation of microenvironments, such as temperature, $\mathrm{pH}$, mechanical stimuli and growth factor gradient can be achieved by microfluidic platform, which will be useful in identifying factors involved in stem cell ${ }_{65}$ proliferation and differentiation ${ }^{9}$. Recently, a gradientgenerating microfluidic platform has been applied to study the effects of a continuous gradient of epidermal growth factor, fibroblast growth factor 2, and platelet-derived growth factor on the growth and differentiation of neural stem cells ${ }^{10}$. 70 Interestingly, colony formation of murine embryonic stem cells can be influenced by different flow rates generated by microfluidic platform although the mechanism behind the flow rate dependent effects remains uncertain ${ }^{11}$. Furthermore, temperature gradient created by microfluidic platform 75 revealed that the importance of temperature on development of Drosophila embryo ${ }^{12}$. Cell fusion between mouse embryonic stem cells and mouse embryonic fibroblasts was 
demonstrated with a newly design microfluidic device ${ }^{13}$. Microfluidic platform has been also shown to facilitate 80 individual gene expression profiling on neural stem cells ${ }^{14,15}$. To further improve the capability of microfluidic platform, 3D microfluidic cell culture systems have been developed, which offers a similar in vitro 3D microenvironment as in vivo ${ }^{16,17}$ Clearly, microfluidic platform has become a new tool for stem ${ }_{85}$ cell and developmental biology research from 2D to 3D culture because it offers a wide range of micro-environmenta control and it is a powerful tool to probe the separate effects of environmental and spatial conditions on cell fate ${ }^{9}$.

In most of the previous experimental investigations on EB 90 differentiation, EBs were only subjected to a single differentiation condition ${ }^{18-21}$. Here we demonstrated that by independently cultivating the two halves of an EB in two separate media resulting from laminar co-flow in a microchannel, cell differentiation can be induced on a half of 95 the EB while the other half of the EB maintained in uninduced stages can be achieved.

\section{Materials and Methods}
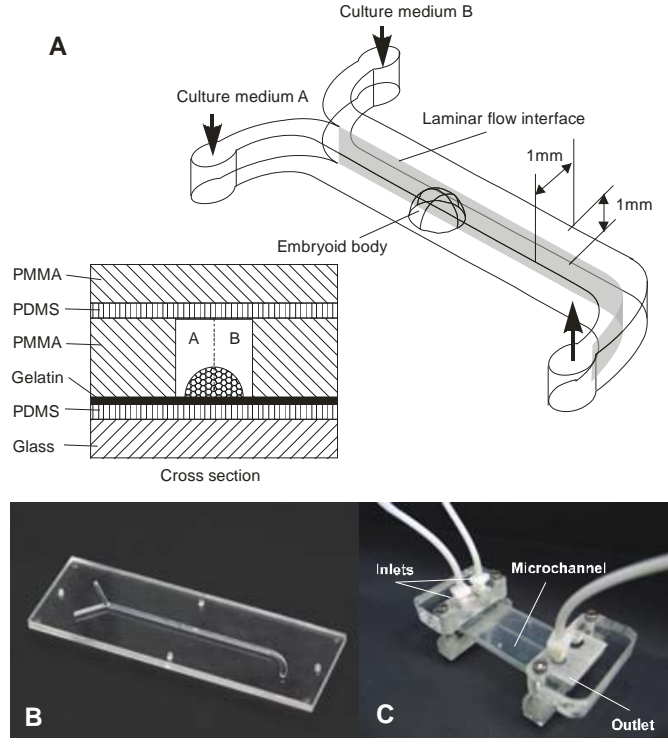

Fig. 1 Schematic diagram illustrating the components of the microfluidic device (A). Photographs of the microfluidic chip (B) and the assembled microfluidic device (including holders, microfluidic chip and tubings) (C).

\section{Mouse ES cell culture}

E14 Mouse ES (mES) cells were cultured in dishes coated 100 with $0.1 \% \mathrm{w} / \mathrm{v}$ gelatin (Sigma) and maintained in a complete medium consisting of Dulbecco's Modified Eagle's Medium (DMEM; Gibco) containing 20\% fetal bovine serum (FBS; Hyclone), 0.1mM 2-mercaptoethanol (Sigma), 1\% MEM nonessential amino acids (Gibco), $100 \mathrm{U} / \mathrm{ml}$ penicillin

105 (Invitrogen), $100 \mathrm{U} / \mathrm{ml}$ streptomycin (Invitrogen) and 1000 $\mathrm{U} / \mathrm{ml}$ ESGRO-LIF (Chemicon), in a humidified incubator $\left(37^{\circ} \mathrm{C}\right.$ in an atmosphere of $\left.5 \% \mathrm{CO}_{2}\right)$, with the medium changed every 2 days. To induce EB formation, mES cells were dissociated into a single-cell suspension by $0.05 \%$ 110 trypsin and $0.04 \%$ EDTA in PBS and plated onto nonadherent bacterial culture dishes and maintained in the complete DMEM medium without LIF for 3 days on a 3D shaking platform in a humidified incubator $\left(37^{\circ} \mathrm{C}\right.$ in an atmosphere of $5 \% \mathrm{CO}_{2}$ ).

\section{Induction of cell differentiation}

For the differentiation assay of EB, a three-day EB grown in bacterial culture dish were plated onto $60 \mathrm{~mm}$ tissue culture dishes coated with $0.5 \% \mathrm{w} / \mathrm{v}$ gelatin and maintained in the complete DMEM medium without LIF in a humidified 20 incubator $\left(37^{\circ} \mathrm{C}\right.$ in an atmosphere of $\left.5 \% \mathrm{CO}_{2}\right)$. After two days incubation, the EB attached and spread onto the tissue culture dishes, and then medium was changed into either differentiation medium [complete L15 medium (Gibco) added with $10 \mu \mathrm{M}$ retinoic acid (RA; Sigma)], or fresh complete L15 125 medium in a humidified incubator $\left(37^{\circ} \mathrm{C}\right)$ with medium changed every 2 days. After 5 days incubation, the EB was then collected and lysed with lysis buffer (PIERCE). 20 $\mu \mathrm{g}$ of EB lysate (5-10 EBs) was used in each lane of western blot analysis.

\section{${ }_{130}$ Western blot analysis}

Mouse anti-PCNA (Santa Cruz), mouse anti-neurofilament 68kDa (NF68) (Abcam), and mouse anti-GAPDH (Santa Cruz) antibodies were used in these experiments. Total proteins were extracted with M-PER mammalian protein extraction 135 reagent (Thermo Scientific). 20 $\mu$ g of EB lysate (5-10 EBs) was loaded on each lane and then separated by $12 \%$ SDSPAGE and transferred onto a nitrocellulose membrane (Whatman). The membranes were incubated with antibodies to specific protein followed by incubation with secondary 140 antibodies: polyclonal goat anti-mouse immunoglobulins/HRP (Dako), polyclonal mouse anti-goat immunoglobulins/HRP (Dako) or polyclonal rabbit anti-goat immunoglobulins/HRP (Dako), and developed with chemiluminescence reagent (Thermo Scientific).

\section{${ }_{145}$ Fabrication of the PMMA/PDMS microfluidic platform}

A Y-shape channel was cut on a rectangular $(76.2 \times 25.4 \mathrm{~mm})$ piece of polymethylmethacrylate (PMMA). A $\mathrm{CO}_{2}$ laser machining system (Universal M-300 Laser Machining Platform, Universal Laser Systems Inc., Arizona, USA) was 150 used for cutting the 1 mm-thick PMMA sheet (Goodfellow, England). Two layers of polydimethylsiloxane (PDMS) at the top and at the bottom of the $\mathrm{Y}$ shape channel were used for sealing of the device (Figure 1A). The PDMS sheet with the thickness of $0.5 \mathrm{~mm}$ was prepared by spin coating the PDMS 155 mixture (Dow Corning Corporation, USA) at $500 \mathrm{rpm}$ for 30 seconds. The prepolymer and the curing agent of PDMS were mixed with a weight ratio of 10:1. The thin PDMS layer was then cured in an oven for 4 hours at $90^{\circ} \mathrm{C}$. Holes were cut in the top layer of PMMA and PDMS to provide inlet and outlet ${ }_{160}$ for the media. The microfluidic chip consisted of two PMMA layers (1mm thick), two layers of PDMS $(0.5 \mathrm{~mm}$ thick) and one glass slide. Thus, the internal dimension (width and 

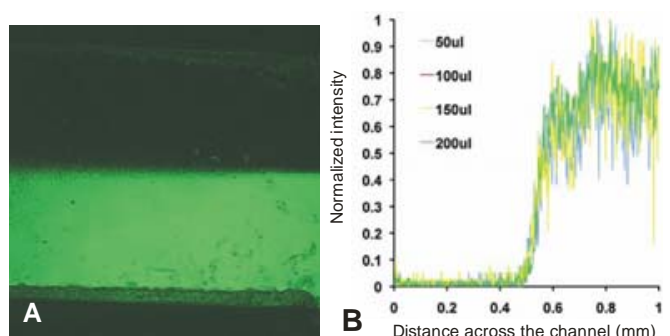

Fig. 2 Measuring the mixing effect of two media in the microchannel different flow rates. The laminar flow generated by different flow rates in the microchannel was visualized by addition of a green fluorescence dye the microchannel was visualized by addition of a green fluorescence dye
(Alexa 488) into one of the media (A). Flow rates of $50 \mu \mathrm{l} / \mathrm{min}$, $100 \mu \mathrm{l} / \mathrm{min}, 150 \mu \mathrm{l} / \mathrm{min}$, and $200 \mu \mathrm{l} / \mathrm{min}$ were examined. The normalized fluorescence intensities across the microchannel were measured and plotted against the width of the microchannel (B). The results showed that all the flow rates tested could maintain a parallel laminar flow.

height) of the microchannel was $1 \mathrm{~mm} \times 1 \mathrm{~mm}$. The inlet and outlet of the channel were cut as a "Y" and a "J" shape for 165 connecting to the inlets and outlet of the holder (Figure 1B) respectively. Two holders were used to fasten the microfluidic chip by screws at both ends. The PMMA holder with sealing rings for the inlets of the microfluidic device was connected to a peristaltic pump (Cole Parmer, USA) by tubing with 0.5 $170 \mathrm{~mm}$ inner diameter (Cole Parmer, USA). Whereas other holde served as outlet, which was connected to a waste bottle (Figure 1C).

Measuring the laminar flow generated by different flow rates in the microfluidic channel

175 Two different media were prepared. Medium A contains complete L15 with a green fluorescence dye (Alexa 488). Medium B contains complete L15 without the green fluorescence dye (Alexa 488). Subsequently, medium A and B were pumped into the microfluidic device at the same flow rate. The ${ }_{80}$ fluorescence intensity of the medium passing through the microfluidic device was imaged by a coupled charge device (CCD) camera attached to the fluorescence microscope at 1 second interval. Flow rates of $50 \mu \mathrm{l} / \mathrm{min}, 100 \mu \mathrm{l} / \mathrm{min}, 150 \mu \mathrm{l} / \mathrm{min}$, and $200 \mu \mathrm{l} / \mathrm{min}$ were examined. The fluorescence intensity across

185 the microchannel (Figure 2A) was measured using the line-scan function of the Zeiss Axiovision software and plotted as a X-Y scatter graph shown in Figure 2B. At $50 \mu \mathrm{l} / \mathrm{min}$, the estimated Reynolds number and Peclet number are on the order of 1 and 1000. Thus flow is laminar, but advection dominates over 190 diffusion. The culture media do not mix across their interface.

\section{Immunofluorescence staining of EBs}

After 5 days incubation, the microfluidic device was dissembled and the EB was fixed with $4 \%$ (w/v) paraformaldehyde for $30 \mathrm{~min}$ and then permabilized with 195 0.5\% Triton X-100 (Amersham) in phosphate buffered saline (PBS) for $30 \mathrm{~min}$. The EB was blocked with $5 \%$ bovine serum albumin (BSA) in PBS for $30 \mathrm{~min}$ and sequentially exposed to primary and secondary antibodies: mouse anti neurofilament $160 \mathrm{kDa}$ (NF160) protein antibodies (Chemicon; 1/1000 200 dilution; room temperature $1 \mathrm{hr}$ ), rabbit anti mouse Ki67 antibodies (Novacastra; 1/500 dilution; room temperature 1 hr), Hexa Fluro 488 goat anti-mouse IgG (Invitrogen; 1/1000 dilution; room temperature $1 \mathrm{hr}$ ), Alexa Fluro 594 goat antirabbit IgG (Invitrogen; 1/1000 dilution; room temperature 1 $205 \mathrm{hr}$ ). Cell nuclei were then counter-stained with DAPI and the coverslip was mounted with ProLong ${ }^{\circledR}$ Gold antifade reagent (Invitrogen). Fluorescent images were obtained by an inverted microscope equipped with motorized stage (Carl Zeiss).

\section{Results and Discussions}

${ }_{210}$ Determination of the minimum flow rate of the medium

To optimize the parallel laminar flow with minimum consumption of the culturing medium, different flow rates were examined. It was found that all the tested flow rates could maintain a parallel laminar flow without intermixing 15 between the fluorescence labelled medium and the nonlabelled medium (Figure 2A and B). Hence, a flow rate of $50 \mu \mathrm{l} / \mathrm{min}$ was chosen in the subsequent experiments to minimize the consumption of the culturing medium.

\section{Biocompability of PDMS for the growth and differentiation of} 220 embryoid bodies

The microfluidic chip was assembled as shown in Figure 1 to test the ability of PDMS to support the growth and differentiation of the embryoid body. A three-day EB (Figure 3Aa) was transferred by a pipette tip into the microchannel, 225 and the whole microfluidic chip was immersed in the complete DMEM in a $100 \mathrm{~mm}$ tissue culture dish. The EB was incubated at $37^{\circ} \mathrm{C}$ for another 2 days (Figure $3 \mathrm{Ab}$ ). When the EB was attached onto the PDMS layer of the microfluidic chip, another two layers of PDMS and PMMA were placed on the 230 PMMA layer as illustrated in Figure 1. A peristaltic pump was connected to the two inlets of the microchannel, which provided a continuous flow of either complete L-15 medium (Gibco), to maintain the normal growth of EB, or complete L15 medium with $10 \mu \mathrm{M}$ RA, to induce cell differentiation of ${ }_{235} \mathrm{~EB}$, at a flow rate of $50 \mu \mathrm{l} / \mathrm{min}$, respectively. The microfluidic device was then incubated in the live imaging system (Carl Zeiss). The growth of EB was monitored by time-lapse microscopy (Carl Zeiss) (Figure 3B). After 5 day of incubation at $37^{\circ} \mathrm{C}$, the microfluidic device was disassembled 240 and the EB in the microchannel was analyzed by immunofluorescence staining using antibodies against Ki67 as proliferation marker and NF160 as cell differentiation marker. It was found that there was strong Ki67 staining on the EB incubated with complete L15 medium, suggesting that growth 245 of EB was not affected by PDMS. Most importantly, addition of RA induced cell differentiation of EB in the microfluidic channel as suggested by the positive immunostaining of NF160 (Figure 3C). Taken together, PDMS has no adverse effect on the growth and differentiation of EB.

250 To investigate the effects of RA on changes in rate of cell proliferation and induction of cell differentiation during EB development, western blot analysis was performed. Our results showed that the level of PCNA, the proliferation marker, in EBs cultured in complete L15 medium was higher 255 than those in complete L15 medium with $10 \mu \mathrm{M}$ RA after 5 days of incubation (Figure 3D and $3 \mathrm{E}$ ) (The two-tailed $\mathrm{P}$ value 


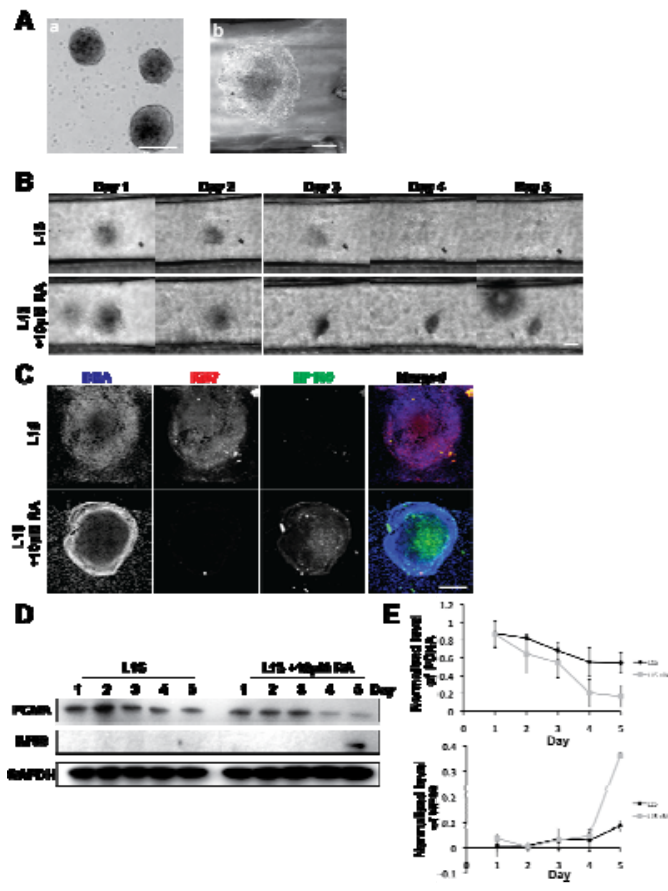

Fig. 3 Culturing of the embryoid bodies in the microfluidic device Optical micrograph of EBs was taken from the non-adherent bacterial culture dish after three days incubation (Aa). A 3-day EB was transferred into the microchannel and cultured with complete L15 medium without LIF for two days in a humidified incubator. Bright field micrograph of the EB was attached onto the surface of the microchannel after incubation (Ab). The microfluidic device was assembled and connected with a peristaltic pump. The EB was then maintained in the microfluidic device under a constant flow rate $(50 \mu \mathrm{l} / \mathrm{min})$ with complete L15 medium or complete $\mathrm{L} 15$ medium with addition of $10 \mu \mathrm{M}$ RA for 5 days. Time-lapse images of EBs cultured in the microfluidic device from day 1 to day 5 images of EBs cultured in the microfluidic device from day 1 to day RA respectively (B). After 5 days incubation, EB in the microchannel cultured with complete L15 medium or complete L15 medium with $10 \mu \mathrm{M}$ RA were fixed and stained with anti-NF 160 and anti-Ki67 antibodies, cell nuclei were counter-stained with DAPI (C). The expression of NF 160 was only detected on the EB maintained with complete L15 medium with $10 \mu \mathrm{M}$ RA. Scale bar, $200 \mu \mathrm{m}$. EBs maintained with complete L15 and complete L15 with $10 \mu \mathrm{M}$ RA from

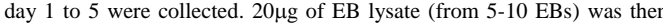
analysed by western blot analysis using antibodies specific for PCNA and NF68 using GAPDH as control (D). Expression level of PCNA and NF68 were quantified and then normalized by level of GAPDH at the same point. The average normalized expression levels of PCNA and NF68 were then plot against number of incubation days. Data are mean \pm standard deviation of four independent western blot analyses (E).

equals 0.0040. By conventional criteria, this difference is considered to be statistically significant). Besides, NF68, which are intermediate filaments found specifically in neurons,

260 was only detected in the EBs maintained in complete L15 medium with $10 \mu \mathrm{M}$ RA for 5 days (Figure 3D and 3E). These results suggested that addition of RA is proficient and effective to induce cell differentiation and slow down the rate of cell proliferation on EB in the microfluidic device.
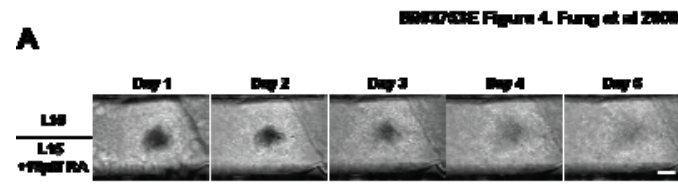

$\mathbf{B}$

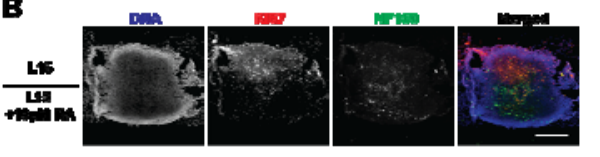

c

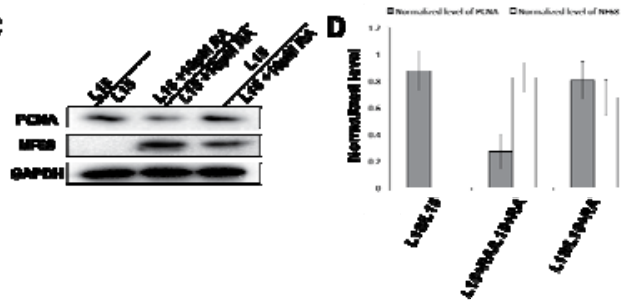

Fig. 4 Induction of neural differentiation on half of EB in the microfluidic device. Time-lapse images of EB cultured under the laminar flow of complete L15 medium and complete L15 with $10 \mu \mathrm{M}$ RA at a constant flow rate of $50 \mu \mathrm{l} / \mathrm{min}$ (A). After 5 days incubation, the $\mathrm{EB}$ in the microchannel was fixed and stained with anti-NF 160 and anti-Ki67 antibodies, cell nuclei were counter-stained with DAPI (B). The expression of NF 160 was detected mainly at the half of EB that was cultured with complete L15 medium with $10 \mu \mathrm{M}$ RA. While strong staining of Ki67 was observed on the half of EB maintained with complete L15 medium (scale bar: $200 \mu \mathrm{m}$ ). For Western blot analysis, three-day EBs were transferred onto the microchannel, the microfluidic device was assembled and then the EBs were maintained with supplying of complete L15 medium, complete L15 medium with $10 \mu \mathrm{M}$ RA or under the laminar flow of complete L15 medium and complete L15 with $10 \mu \mathrm{M}$ RA at a constant flow rate respectively. After 5 days of incubation,

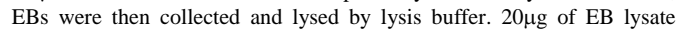
(from 5-10 EBs) was then analysed western blot analysis using antibodies specific for PCNA and NF 68 with GAPDH as control (C). Expression level of PCNA and NF68 were quantified and then normalized by level of GAPDH. Data are mean \pm standard deviation of four independent western blot analyses (D).

Induction of neural differentiation on half of the EB while maintaining the other half in un-induced stage

We then tested the idea whether the microfluidic device could be used to induce cell differentiation on half of the EB 270 while maintaining the rest in un-induced stages, a three-day EB was seeded into the microfluidic device as mentioned above. The EB were supplied with culturing media via the two inlets of the microfluidic device at the flow rate of $50 \mu \mathrm{l} / \mathrm{min}$ each. One stream consists of complete L-15 medium to 275 maintain the normal growth of EB. The other stream consists of complete L-15 medium with $10 \mu \mathrm{M}$ RA, to induce cell differentiation of EB. The microfluidic device was incubated in the live imaging system (Carl Zeiss) at $37^{\circ} \mathrm{C}$. The growth of EB was monitored by time-lapse microscopy (Carl Zeiss) 280 (Figure 4A). At the end of the experiment, the EB was analyzed by immunofluorescence staining using antibodies against Ki67 as proliferation marker or NF160 as cell differentiation marker. 
A red fluorescence dye was added into one of the media to 285 further confirm that the laminar flow was not disturbed during the incubation. The fluorescence intensity across the channel was measured (data not shown). Neurofilament (NF160) was only found in one half of the EB, while the other half has strong staining of Ki67 (Figure $4 \mathrm{~B}$ and Electronic

290 Supplementary Information Figure 1). Consistently, we found that the expression of NF68 was present in the EB supplied with complete L15 medium with $10 \mu \mathrm{M}$ RA and the EB under the laminar flow of complete L15 medium and complete L15 medium with $10 \mu \mathrm{M}$ RA (Figure 4C and 4D). Most

295 importantly, the level of PCNA for the EBs cultivated under the laminar flow of complete L15 medium and complete L15 medium with $10 \mu \mathrm{M}$ RA (L15/L15+RA) is significantly higher than those cultivated in complete L15 medium (L15/L15) and complete L15 medium with $10 \mu \mathrm{M}$ RA 300 (L15+RA/L15+RA) (Figure 4C and 4D) (The two-tailed $\mathrm{P}$ value equals 0.0014 By conventional criteria, this difference is considered to be statistically significant), suggesting portion of the EB remains un-induced and proliferative.

\section{Conclusions}

305 In conclusion, we have fabricated a microfluidic device and cultured EB derived from mouse ES cells in this device. Furthermore, we have applied the microfluidic device in EB differentiation, resulting in differentiated and un-induced cells in different areas of the same EB simultaneously. Specific 310 differentiation into different specialized cell lineages on the same EB can be achieved by applying microfluidic technology.

\section{Acknowledgement}

315 This work was supported by Biomedical Research Council, A*STAR, Singapore (05/1/22/19/388, 08/1/22/19/568), Academic Research Council, Ministry of Education, Singapore (RG37/08 and RG22/05).

\section{References}

320 1. J. A. Thomson, J. Itskovitz-Eldor, S. S. Shapiro, M. A. Waknitz, J. J. Swiergiel, V. S. Marshall and J. M. Jones, Science, 1998, 282, 11451147.

2. J. S. Odorico, D. S. Kaufman and J. A. Thomson, Stem Cells, 2001, 19, 193-204.

325 3. G. M. Keller, Curr Opin Cell Biol, 1995, 7, 862-869.

4. J. Itskovitz-Eldor, M. Schuldiner, D. Karsenti, A. Eden, O. Yanuka, M. Amit, H. Soreq and N. Benvenisty, Mol Med, 2000, 6, 88-95.

5. D. Choi, H. J. Lee, S. Jee, S. Jin, S. K. Koo, S. S. Paik, S. C. Jung, S. Y. Hwang, K. S. Lee and B. Oh, Stem Cells, 2005, 23, 817-827.

330 6. I. Desbaillets, U. Ziegler, P. Groscurth and M. Gassmann, Exp Physiol, 2000, 85, 645-651.

7. M. J. Shamblott, J. Axelman, J. W. Littlefield, P. D. Blumenthal, G. R. Huggins, Y. Cui, L. Cheng and J. D. Gearhart, Proc Natl Acad Sci U S A, 2001, 98, 113-118.

335 8. G. M. Whitesides, Nature, 2006, 442, 368-373.

9. D. van Noort, S. M. Ong, C. Zhang, S. Zhang, T. Arooz and H. Yu, Biotechnol Prog, 2009, 25, 52-60.
10. B. G. Chung, L. A. Flanagan, S. W. Rhee, P. H. Schwartz, A. P. Lee, E. S. Monuki and N. L. Jeon, Lab Chip, 2005, 5, 401-406.

340 11. L. Kim, M. D. Vahey, H. Y. Lee and J. Voldman, Lab Chip, 2006, 6, 394-406.

12. E. M. Lucchetta, J. H. Lee, L. A. Fu, N. H. Patel and R. F. Ismagilov, Nature, 2005, 434, 1134-1138.

13. A. M. Skelley, O. Kirak, H. Suh, R. Jaenisch and J. Voldman, Nat Methods, 2009, 6, 147-152.

14. Y. Chen and J. F. ZhongR, Methods Mol Biol, 2008, 438, 293-303.

15. J. F. Zhong, Y. Feng and C. R. Taylor, Curr Med Chem, 2008, 15, 2897-2900.

16. Y. C. Toh, S. Ng, Y. M. Khong, V. Samper and H. Yu, Assay Drug Dev Technol, 2005, 3, 169-176.

17. Y. C. Toh, C. Zhang, J. Zhang, Y. M. Khong, S. Chang, V. D. Samper, D. van Noort, D. W. Hutmacher and H. Yu, Lab Chip, 2007, 7, 302-309.

18. H. Wichterle, I. Lieberam, J. A. Porter and T. M. Jessell, Cell, 2002, 110, 385-397.

19. J. Rohwedel, V. Maltsev, E. Bober, H. H. Arnold, J. Hescheler and A. M. Wobus, Dev Biol, 1994, 164, 87-101.

20. O. Brustle, K. N. Jones, R. D. Learish, K. Karram, K. Choudhary, O. D. Wiestler, I. D. Duncan and R. D. McKay, Science, 1999, 285, 754756.

21. G. Balconi, R. Spagnuolo and E. Dejana, Arterioscler Thromb Vasc Biol, 2000, 20, 1443-1451.

This journal is (c) The Royal Society of Chemistry [year] 
Formatted: E2 References, Tab stops:

Not at $12.23 \mathrm{~cm}$ 